\title{
Near-Field Imaging of Cell Membranes in Liquid Enabled by Active Scanning Probe Mechanical Resonance Control
}

\author{
Kyoung-Duck Park, ${ }^{1,2}$ Markus B. Raschke, ${ }^{1}$ Min Jung Jang, ${ }^{3}$ Jung Hwa \\ Kim, ${ }^{3}$ Beom-Hoan O, ${ }^{2}$ Se-Geun Park, ${ }^{2}$ El-Hang Lee, ${ }^{2}$ and Seung Gol Lee ${ }^{2}$ \\ ${ }^{1}$ Department of Physics, Department of Chemistry, \\ and JILA, University of Colorado, Boulder, CO, 80309, USA \\ ${ }^{2}$ Department of Information and Communication Engineering, \\ Inha University, Incheon, 402-751, Republic of Korea \\ ${ }^{3}$ Department of Biological Sciences, Inha University, \\ Incheon, 402-751, Republic of Korea
}

(Dated: July 27, 2016) 


\section{MODELING OF HIGH-Q OSCILLATOR}

The vibration behaviors of high-Q oscillator are numerically analyzed by modeling with five vibration sections (see Fig. S1). The variation of resonance frequency and Q-factor are calculated as a function of lengths $l_{1}$ and $l_{2}\left(l_{1}+l_{2}\right.$ is maintained to $\left.14.0 \mathrm{~mm}\right)$ for three different lengths of the protruded tip $\left(l_{3}=1.5,2.0\right.$, and $\left.3.0 \mathrm{~mm}\right)$ to understand the influence of tip length to the resonance characteristics of oscillator (see Fig. S2). While three oscillators have different resonance frequencies, variation tendencies of them are not significantly different and their Q-factors are almost same. From these vibration behaviors, we make a conclusion that the resonance condition is dominantly determined by the lengths $l_{1}$ and $l_{2}$, but nearly irrespective to the $l_{3}$. However, the tip length $\left(l_{3}\right)$ is significant when it is dipped into the liquid because the dithering amplitude of tip is dramatically changed with respect to $l_{3}$ and it affects to the Q-factor of oscillator in liquid.

\section{SUPPLEMENT DISCUSSION OF FIG. 3}

Measured periodic variations of resonance characteristics in Fig. 3a are nearly same irrespective of the environments. This result means $l_{2}$ is the still dominant factor to determine resonance condition even when the tip is partially dipped into a liquid, i.e., the resonance condition in liquid environment is not significantly different from that in air. The Q-factor also show similar variation tendency though the Q-factor in water is relatively decreased due to the water viscosity.

It should be also noted that the experimentally observed discrepancy of the resonance frequency and the Q-factor between $l_{2}=\sim 3 \mathrm{~mm}$ and $l_{2}=\sim 9 \mathrm{~mm}$ is attributed to the relatively different compressive force at the pin point, which depends on the position of knife edge. That is to say, despite the compressive force of the knife edge is held constant, the compressive force of the pin point is slightly varied depend on the position of knife edge as described in Fig. S4b. This effect makes systematic increase of resonance frequency and Q-factor as a function of $l_{2}$.

From the theory and experiment results, it is concluded that the $l_{2}$ should be $(2 n-1) \lambda_{F} / 4$ for the efficient coupling and the $l_{3}$ should be $m \lambda_{F} / 2$ for the tip-end to be placed at a node of $l_{3}$ vibration, where $n$ and $m$ are integers independent of each other. 


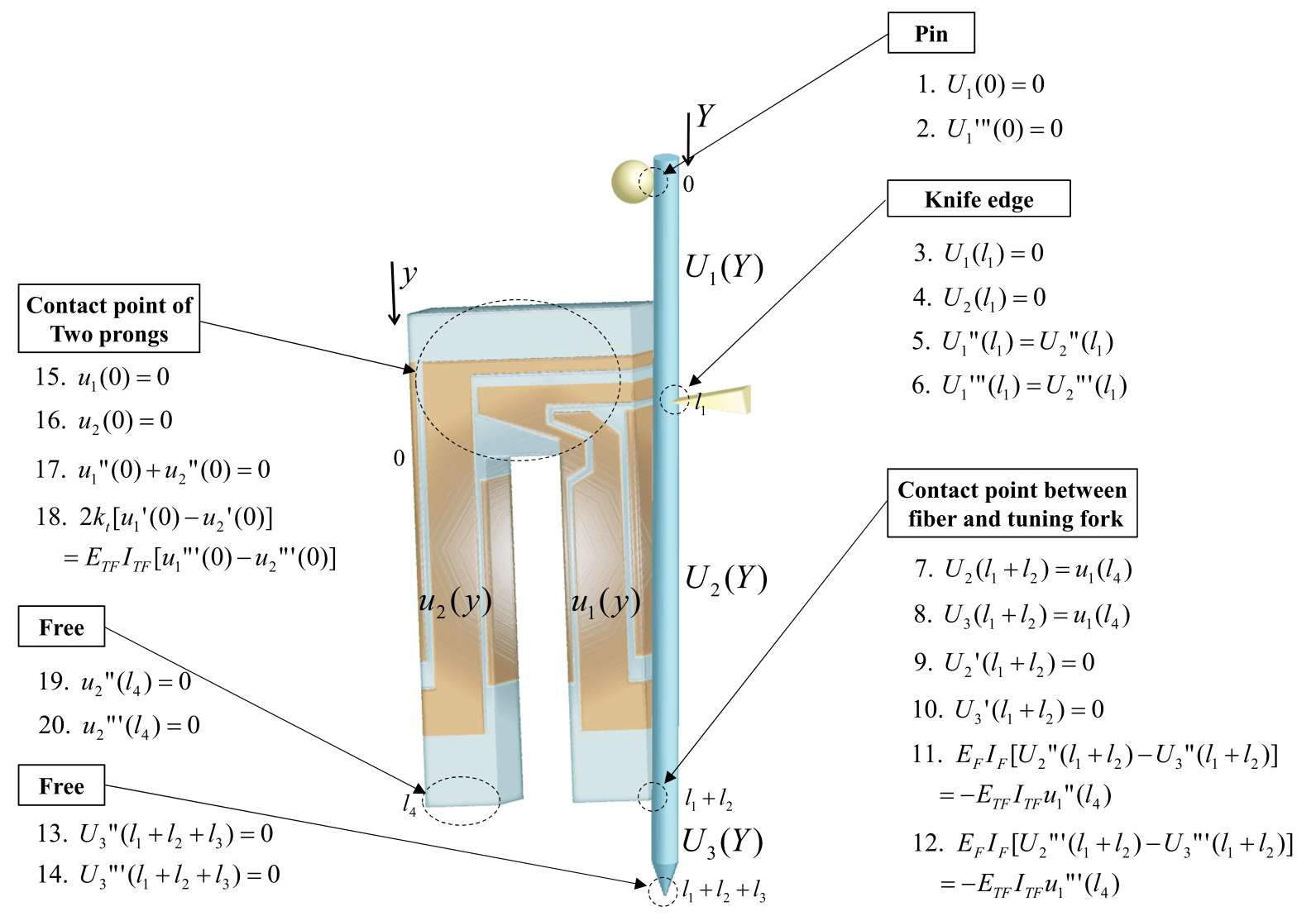

Figure S1. Modeling of the tuning fork/fiber probe coupled oscillator. A total of 12 boundary conditions are applied at the six vibration points of the five vibration sections. The boundary conditions in the two free parts are deduced based on the bending vibration of beam theory. Other boundary conditions are carefully derived by considering mechanical properties of each part. 


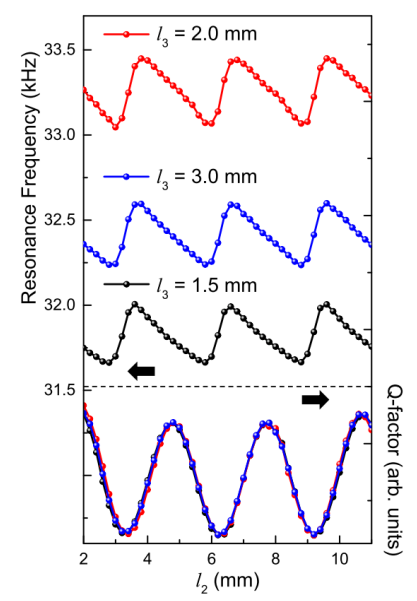

Figure S2. Calculation results of the variation of resonance frequency and Q-factor for three different tip lengths $\left(l_{3}=1.5,2.0\right.$, and $\left.3.0 \mathrm{~mm}\right)$ with respect to the change of $l_{1}$ and $l_{2}\left(l_{1}+l_{2}\right.$ are maintained to $14.0 \mathrm{~mm}$ ). While the resonance frequencies of three NSOM probes are different, the variation is very similar and Q-factors are almost the same.

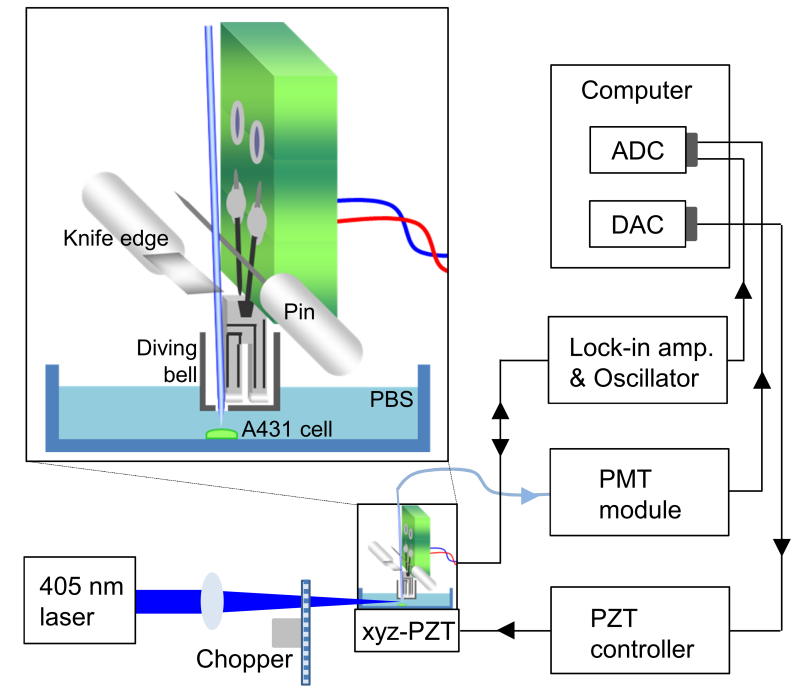

Figure S3. Schematic of the NSOM system. The tuning fork is electrically driven by an oscillator (function generator) and the voltage, which is proportional to the mechanical amplitude of tuning fork, is monitored by a lock-in amplifier. The excitation laser is modulated by a chopper to remove noise signal in PMT detection. The high-Q oscillator and diving bell structure are fixed and the sample (cell and culture dish) is scanned by a xyz PZT. 


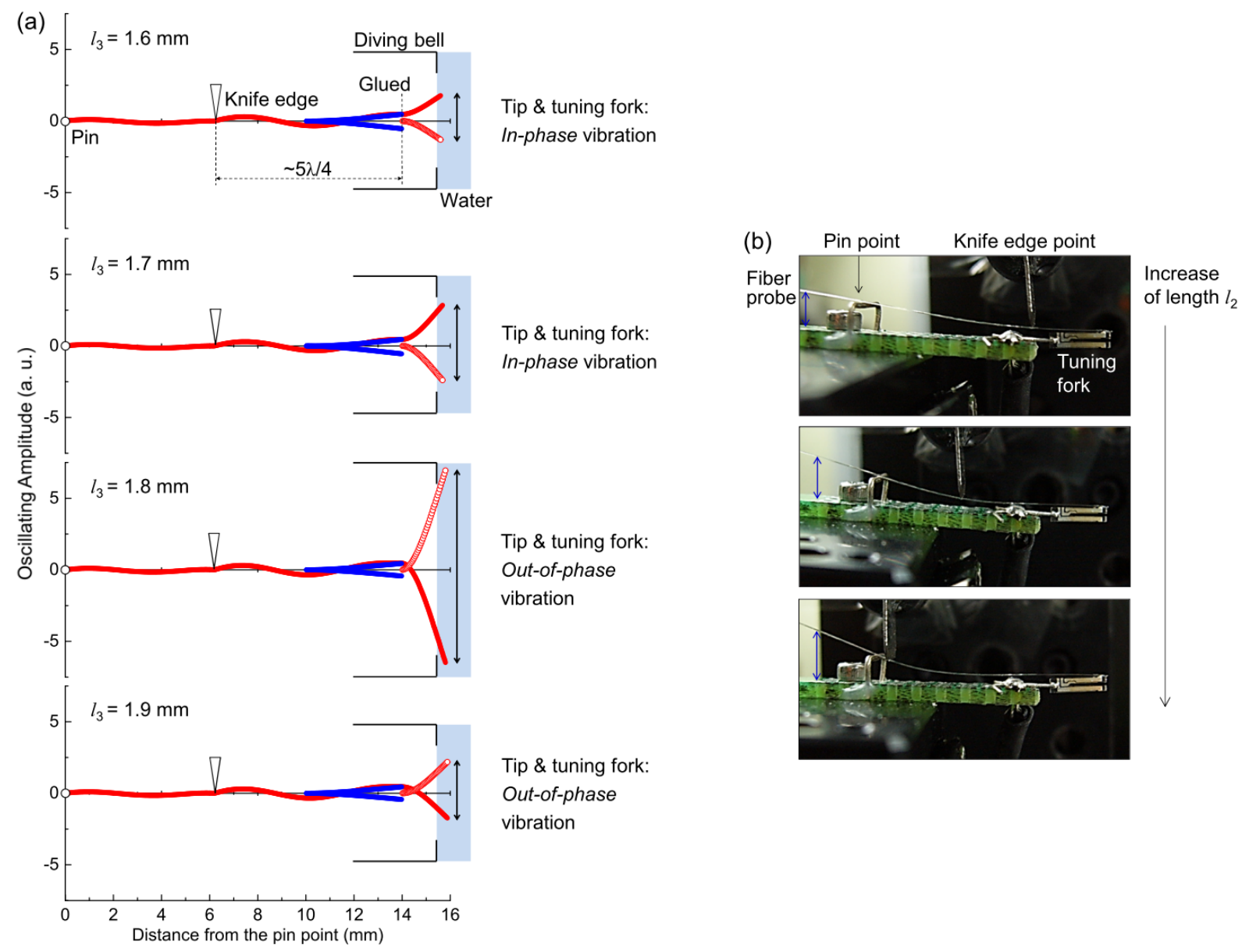

Figure S4. (a) Calculated oscillating amplitude curves of the tuning fork (blue lines) and the fiber probe (red line) as a function of $l_{3}$ while maintaining $l_{1}=6.2 \mathrm{~mm}$ and $l_{2}=7.8 \mathrm{~mm}$. Note that the maximum tip-end amplitude $\left(l_{3}=1.8 \mathrm{~mm}\right)$ is calculated at the first out-of-phase vibration. Because the amplitude of an out-of-phase vibration is generally larger than in-phase vibration, and the tip-end of $l_{3}=1.8 \mathrm{~mm}$ tip is most closed to the antinode position among the out-of-phase vibrations $\left(l_{3} \geq 1.8 \mathrm{~mm}\right.$ ). (b) Photos of test setup for the two nodal wedges method (pin and knife edge positions are not matched with the main text). The experimentally observed discrepancy of the resonance frequency and the Q-factor between $l_{2}=\sim 3 \mathrm{~mm}$ and $l_{2}=\sim 9 \mathrm{~mm}$ (Fig. 2(a)) is attributed to the relatively different compressive force at the pin point, which depends on the position of knife edge. That is to say, despite the compressive force of the knife edge is held constant, the compressive force of the pin point is slightly varied depend on the position of knife edge. This effect makes systematic increase of resonance frequency and Q-factor as a function of $l_{2}$. 
(a) Gold nanoparticles
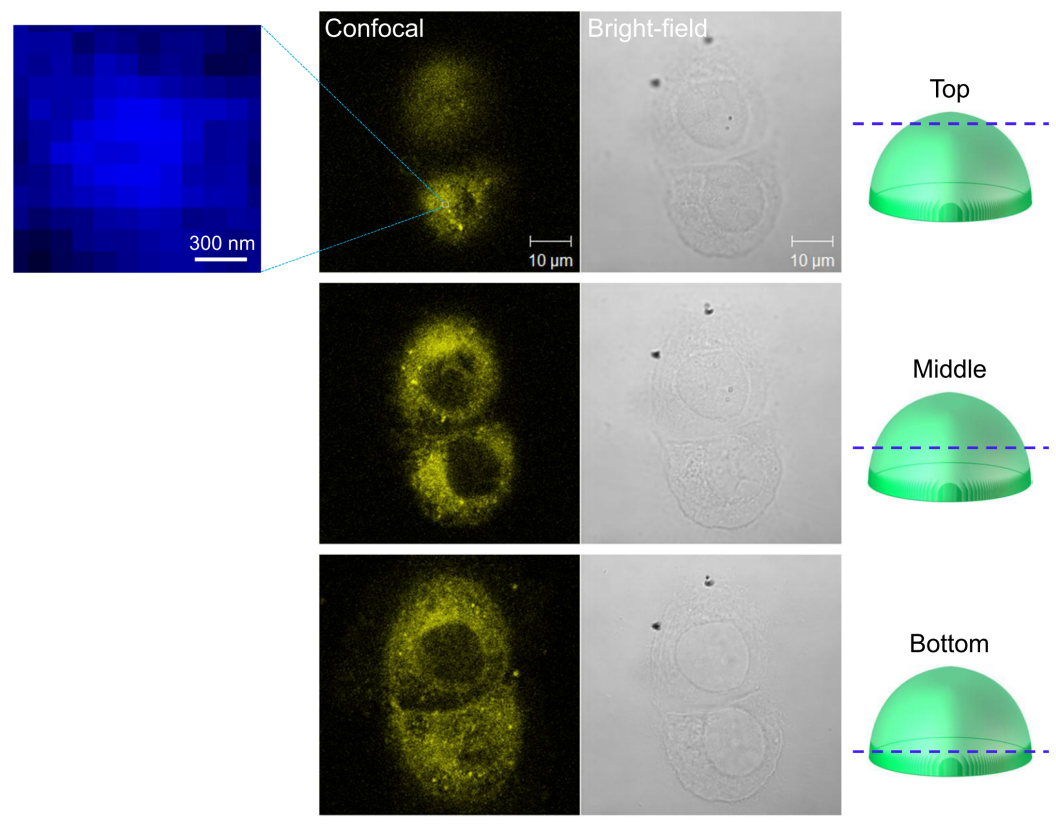

(b) FITC fluorophore
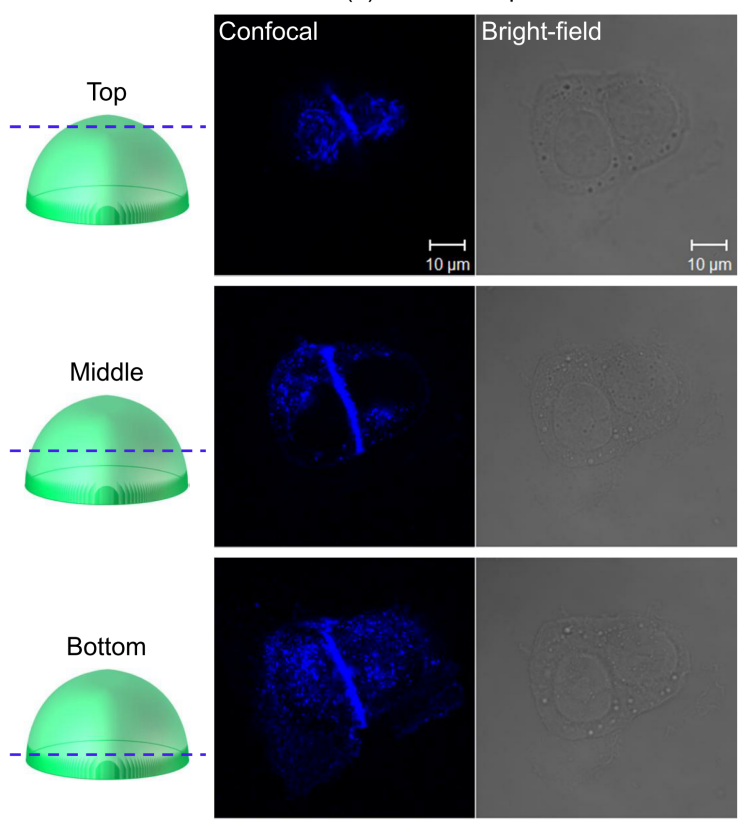

Figure S5. (a) Confocal laser scanning microscope images of the A431 cells, where EGFRs are labeled by (a) AuNPs and (b) FITC fluorophores. A $405 \mathrm{~nm}$ laser is used for measuring scattering (of AuNPs) or fluorescence (of FITC fluorophores) signals of the A431 cells. The EGFR imaging is done with a 100x magnification, 1.3 NA oil immersion objective lens by changing a focal plane from top to bottom of the cell. The left zoomed-in image is used in Fig. 4(e) to compare spatial resolution with NSOM image. 


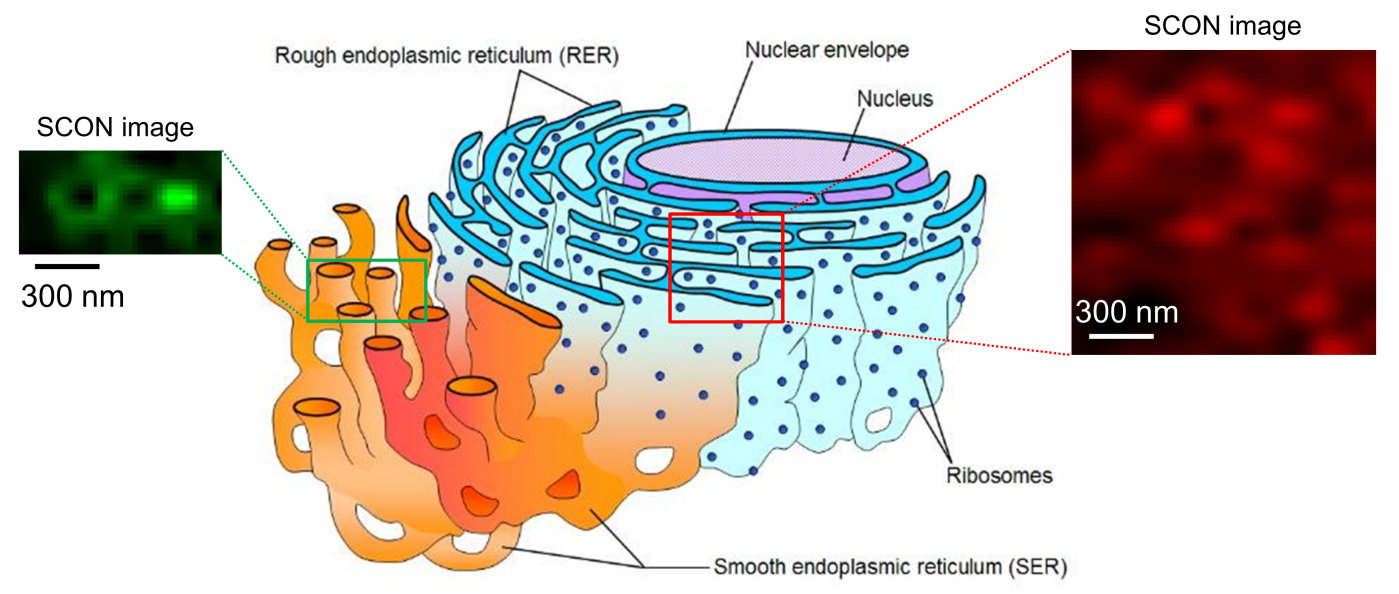

Figure S6. Cartoons of organelle structures in the cells. The endoplasmic reticulum (ER) forms an interconnected network of flattened, membrane-enclosed sacs or tubes known as cisternae. It is known that the membranes of ER are continuous with the outer membrane of the nuclear envelope. There are two types of ER: rough ER and smooth ER. The main function of rough ER is the synthesizing and transporting proteins between the nucleus and cell-membrane, and those proteins highly interact with EGFRs. The structure of rough ER is known to be folded like a pile of folded papers. Therefore, we believe the red image zoomed in the left bottom inset is the rough ER region. On the other hand, the observed nanoscale mesh like distribution (green image) is matched with the well known structure of smooth ER: tube shaped like a pipeline with many twists and turns. The organelle structure in the figure is taken from Cronodon.com, and is used with the permission of Cronodon.com. 


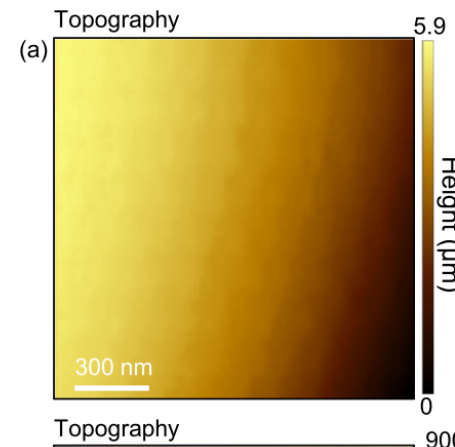

(c)

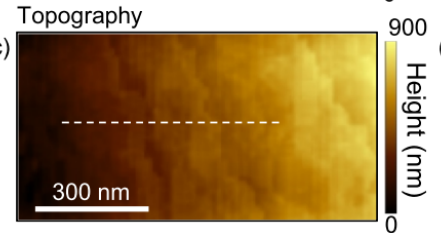

NSOM image

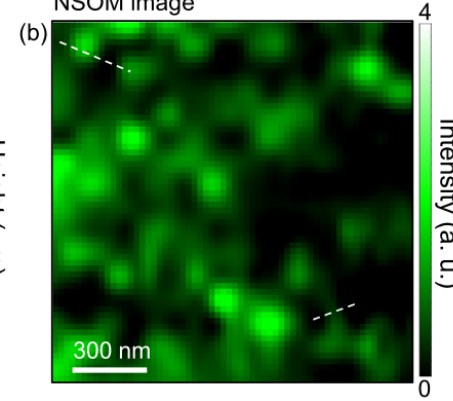

NSOM image

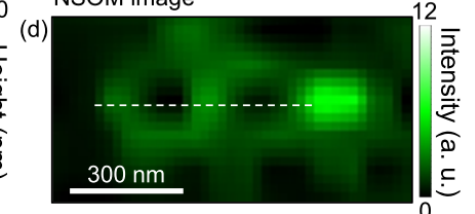

(e)
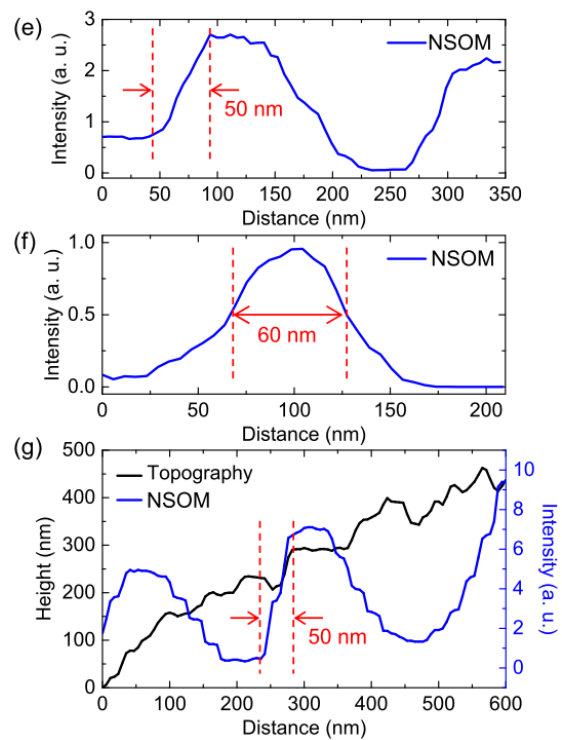

Figure S7. Topographies (a, c) and NSOM images (b, d) of the A431 cell immersed in PBS. (e-g) Line profiles derived from the topography and the NSOM images. The $\sim 50 \mathrm{~nm}$ spatial resolution is confirmed, and the NSOM images are not correlated with the surface structure.

(a)

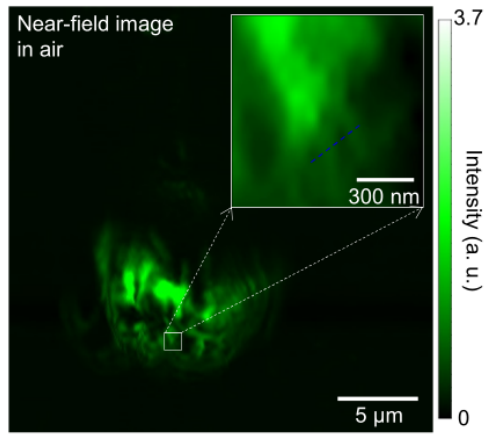

(b)

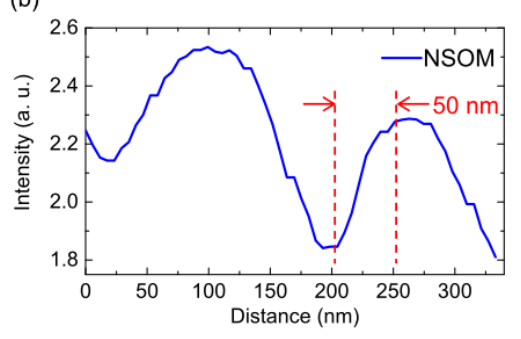

Figure S8. (a) NSOM image of the A431 cell in air after evaporation of PBS. (b) Line profile derived from the NSOM image. Blurred NSOM images are generally repeatable for different cells in air. We believe the blurred images are attributed to the aggregation effect of AuNPs which originated from the structural deformation (inside and outside) of cells in evaporation process. The spatial resolution is same as NSOM images obtained in PBS. 


\section{SCANNING SPEED OF SUPER-RESOLUTION MICROSCOPY}

The slow scan rate and the difficulty of living cell imaging are main shortcomings of both scanning probe methods and far-field super-resolution microscopies. We have compared approximate scan speed of NSOM and conventional methods.

- NSOM: Pixel dwell time of $\sim$ ms due to the decay time of tuning fork.

- STED: Pixel dwell time of $\sim 50 \mu$ s with $\sim 50 \mathrm{~nm}$ spatial resolution, yet the scan rate is much slower to increase resolution [1].

- PALM, STORM: Much slower than NSOM. These methods reconstruct an image from single-molecule fluorescence of repeated measurement. Therefore, long acquisition time is required for sufficient accumulation of a fluorophore localization density. In addition, the rate of accumulating localization points is limited by the switching kinetics of fluorescent probes. E.g., estimation for the imaging speed of PALM: $>9$ h to achieve $\sim 20 \mathrm{~nm}$ resolution for uniformly distributed molecules over a $10 \mu \mathrm{m}$ x $10 \mu \mathrm{m}$ area [2]. Recently, many techniques are being applied to improve scan rate of super-resolution microscopies [3]. To improve scan rate of NSOM several ways can be applied, e.g., phase modulation feedback method [4], using $\mathrm{MHz}$ frequency quartz resonator [5], etc. 
[1] Birka Hein, Katrin I Willig, and Stefan W Hell, "Stimulated emission depletion (sted) nanoscopy of a fluorescent protein-labeled organelle inside a living cell," Proc. Natl. Acad. Sci. USA 105, 14271-14276 (2008).

[2] Samuel T Hess, Thanu PK Girirajan, and Michael D Mason, "Ultra-high resolution imaging by fluorescence photoactivation localization microscopy," Biophys. J. 91, 4258-4272 (2006).

[3] Peter W Winter and Hari Shroff, "Faster fluorescence microscopy: advances in high speed biological imaging," Curr. Opin. Chem. Biol. 20, 46-53 (2014).

[4] AGT Ruiter, JA Veerman, KO van Der Werf, and NF van Hulst, "Dynamic behavior of tuning fork shear-force feedback," Appl. Phys. Lett. 71, 28-30 (1997).

[5] Seiji Heike and Tomihiro Hashizume, "Atomic resolution noncontact atomic force/scanning tunneling microscopy using a 1 mhz quartz resonator," Appl. Phys. Lett. 83, 3620-3622 (2003). 